\title{
RUIN PROBABILITY WITH PARISIAN DELAY FOR A SPECTRALLY NEGATIVE LÉVY RISK PROCESS
}

\author{
IRMINA CZARNA*** AND \\ ZBIGNIEW PALMOWSKI, ${ }^{* * * *}$ University of Wroctaw
}

\begin{abstract}
In this paper we analyze the so-called Parisian ruin probability, which arises when the surplus process stays below 0 longer than a fixed amount of time $\zeta>0$. We focus on a general spectrally negative Lévy insurance risk process. For this class of processes, we derive an expression for the ruin probability in terms of quantities that can be calculated explicitly in many models. We find its Cramér-type and convolutionequivalent asymptotics when reserves tend to $\infty$. Finally, we analyze some explicit examples.
\end{abstract}

Keywords: Lévy process; ruin probability; asymptotics; Parisian ruin; risk process

2010 Mathematics Subject Classification: Primary 60J99; 93E20; 60G51

\section{Introduction}

In risk theory we usually consider the classical Cramér-Lundberg risk process

$$
X_{t}=x+p t-S_{t},
$$

where $x>0$ denotes the initial reserve and

$$
S_{t}=\sum_{i=1}^{N_{t}} U_{i}
$$

is a compound Poisson process. We assume that the $U_{i}, i=1,2, \ldots$, are independent and identically distributed claims (with distribution function $F$ and tail $\bar{F}:=1-F$ ). The arrival process is a homogeneous Poisson process $N_{t}$ with intensity $\lambda$. Premium income is modeled by a constant premium density $p$ and the net profit condition is then $\lambda \nu / p<1$, where $\mathrm{E}\left(U_{1}\right)=$ $v<\infty$. Lately, a more general setting for a spectrally negative Lévy process has been considered. That is, $X=\left\{X_{t}\right\}_{t \geq 0}$ is a process with stationary and independent increments with only negative jumps. We will assume that the process starts from $X_{0}=x$ and later we will use the conventions that $\mathrm{P}\left(\cdot \mid X_{0}=x\right)=\mathrm{P}_{x}(\cdot)$ and $\mathrm{P}_{0}=\mathrm{P}$. Such a process takes into account not only large claims compensated by a steady income at rate $p>0$, but also small perturbations arising from a Gaussian component and possibly in addition (when $\Pi_{X}(-\infty, 0)=\infty$ for a Lévy measure $\Pi_{X}$ of $X$ ) compensated by a countable, infinite number of small claims arriving over each finite time horizon (see, e.g. [15]).

One of the most important characteristics in risk theory is the ruin probability defined by $\mathrm{P}\left(\tau_{0}^{-}<\infty\right)$ for $\tau_{0}^{-}=\inf \left\{t \geq 0: X_{t}<0\right\}$. In this paper we extend this notion to the so-called

* Postal address: Department of Mathematics, University of Wrocław, pl. Grunwaldzki 2/4, 50-384 Wrocław, Poland.

** Email address: czarna@math.uni.wroc.pl

*** Email address: zbigniew.palmowski@gmail.com 
Parisian ruin probability, which occurs if the process $X$ stays below 0 for a longer period than a fixed time $\zeta>0$. Formally, we define the Parisian time of ruin by

$$
\tau^{\zeta}=\inf \left\{t>0: t-\sup \left\{s<t: X_{s} \geq 0\right\} \geq \zeta, X_{t}<0\right\}
$$

and the Parisian ruin probability by

$$
\mathrm{P}\left(\tau^{\zeta}<\infty \mid X_{0}=x\right)=\mathrm{P}_{x}\left(\tau^{\zeta}<\infty\right) .
$$

The $\zeta=0$ case corresponds to the classical ruin problem, which we do not deal with in this paper. The name for this problem is borrowed from the Parisian option. Depending on the type of such an option, the prices are activated or canceled if the underlying asset stays above or below the barrier long enough in a row (see [1], [7], and [10]). We believe that the Parisian ruin probability could be a better measure of risk in many situations, giving insurance companies the chance to achieve solvency. The Parisian ruin probability has already been considered by Dassios and $\mathrm{Wu}$ [8], who found the Parisian ruin probability for the classical risk process (1) with exponential claims and for the Brownian motion with drift. In Dassios and Wu [9], Cramértype asymptotics were found for (1). In this paper, using fluctuation theory, we show that these results could be extended to the case of a general spectrally negative Lévy process. In particular, we show how to establish these results when the process is the sum of an independent classical risk process (1) and Brownian or $\alpha$-stable motion perturbations Additionally, we derive the asymptotics of the Parisian ruin probability in the convolution-equivalent case, that is, when the claim size has a heavy-tailed distribution. Other relevant papers are [19] and [20], where, loosely speaking, the deterministic and fixed delay $\zeta$ is replaced by an independent exponential random variable. Another formula for the Parisian ruin probability was recently also derived in [21].

This paper is organized as follows. In Section 2 we introduce basic notions and notation. In Section 3 we give the main representation of the Parisian ruin probability. In Sections 4 and 5 we give asymptotics of the Parisian ruin probability in the Cramér and convolution-equivalent cases, respectively. Finally, in Section 6 we analyze some particular examples.

\section{Preliminaries}

On $\left(\Omega, \mathcal{F},\left\{\mathcal{F}_{t}\right\}_{\{t \geq 0\}}, \mathrm{P}\right)$ we define the spectrally negative Lévy process $X=\left\{X_{t}\right\}_{t \geq 0}$, that is, a Lévy process with Lévy measure $\Pi_{X}$ satisfying $\Pi_{X}(0, \infty)=0$. Assume that $X_{0}=x>0$ and that $X_{t} \rightarrow \infty$ as $t \rightarrow \infty$ almost surely (a.s.) (that is, reserves of the insurance company increase to $\infty$ a.s.). This assumption excludes the case of a compound Poisson process with negative jumps. We also assume that $\mathrm{E} X_{1}<\infty$. Note that $\mathrm{E} X_{1}>0$ by the drift assumption and Theorem 7.2 of [17]. With $X$ we associate the Laplace exponent $\varphi(\beta):=(1 / t) \log \mathrm{E}\left(\mathrm{e}^{\beta X_{t}}\right)$ defined for all $\beta \geq 0$, and the function $\Phi(q)=\sup \{\theta \geq 0: \varphi(\theta)=q\}$. We will also consider the dual process $\overline{\hat{X}}_{t}=-X_{t}$, which is a spectrally positive Lévy process with Lévy measure $\Pi_{\hat{X}}(0, y)=\Pi_{X}(-y, 0)$. Characteristics of the dual process $\hat{X}$ are denoted with a 'hat' symbol (caret). For the process $X$, we define an ascending ladder process $\left(L^{-1}, H\right)=\left\{\left(L_{t}^{-1}, H_{t}\right)\right\}_{t \geq 0}$ by

$$
L_{t}^{-1}:= \begin{cases}\inf \left\{s>0: L_{s}>t\right\} & \text { if } t<L_{\infty} \\ \infty & \text { otherwise }\end{cases}
$$

and

$$
H_{t}:= \begin{cases}X_{L_{t}^{-1}} & \text { if } t<L_{\infty} \\ \infty & \text { otherwise }\end{cases}
$$


where $L=\left\{L_{t}\right\}_{t \geq 0}$ is a local time at the maximum (see [17, p. 140]). Recall that $\left(L^{-1}, H\right)$ is a bivariate subordinator with Laplace exponent

$$
\kappa(\theta, \beta)=-\frac{1}{t} \log \mathrm{E}\left(\mathrm{e}^{-\theta L_{t}^{-1}-\beta H_{t}} \mathbf{1}_{\left\{t \leq L_{\infty}\right\}}\right)
$$

and Lévy measure denoted by $\Pi_{H}$. We define a descending ladder process $\left(\hat{L}^{-1}, \hat{H}\right)=$ $\left\{\left(\hat{L}_{t}^{-1}, \hat{H}_{t}\right)\right\}_{t \geq 0}$ with the Laplace exponent $\hat{\kappa}(\theta, \beta)$ constructed from the dual process $\hat{X}$. Recall that $\hat{L}_{\infty}$ has an exponential distribution with parameter $\hat{\kappa}(0,0)$. Moreover, from the WienerHopf factorization we have

$$
\kappa(\theta, \beta)=\Phi(\theta)+\beta, \quad \hat{\kappa}(\theta, \beta)=\frac{\theta-\varphi(\beta)}{\Phi(\theta)-\beta} ;
$$

see [17, pp. 169-170]. Hence,

$$
\hat{\kappa}(0,0)=\varphi^{\prime}(0+) .
$$

We introduce a potential measure $\mathcal{U}$ defined by

$$
\mathcal{U}(\mathrm{d} x, \mathrm{~d} s)=\int_{0}^{\infty} \mathrm{P}\left(L_{t}^{-1} \in \mathrm{d} s, H_{t} \in \mathrm{d} x\right) \mathrm{d} t
$$

with Laplace transform $\int_{[0, \infty)^{2}} \mathrm{e}^{-\theta s-\beta x} U(\mathrm{~d} x, \mathrm{~d} s)=1 / \kappa(\theta, \beta)$ and renewal measure

$$
U(\mathrm{~d} x)=\int_{[0, \infty)} U(\mathrm{~d} x, \mathrm{~d} s)=\mathrm{E}\left(\int_{0}^{\infty} \mathbf{1}_{\left\{H_{t} \in \mathrm{d} x\right\}} \mathrm{d} t\right) .
$$

For the spectrally negative Lévy process, the ascending ladder height process is a linear drift and, hence, the renewal measure is just the Lebesgue measure:

$$
U(\mathrm{~d} x)=\mathrm{d} x .
$$

Moreover, considering the renewal measure $\hat{U}(\mathrm{~d} z)$ for $\left(\hat{L}^{-1}, \hat{H}\right)$, from (2) we have

$$
\int_{0}^{\infty} \mathrm{e}^{-\theta z} \hat{U}(\mathrm{~d} z)=\frac{\theta}{\varphi(\theta)}
$$

see $[17$, p. 195].

We will also use the first passage times

$$
\tau_{x}^{-}=\inf \left\{t \geq 0: X_{t}<x\right\}, \quad \tau_{x}^{+}=\inf \left\{t \geq 0: X_{t}>x\right\} .
$$

Suppose now that the probabilities $\left\{\mathrm{P}_{x}\right\}_{x \in \mathbb{R}}$ correspond to the conditional version of $\mathrm{P}$ where $X_{0}=x$ is given. We simply write $\mathrm{P}_{0}=\mathrm{P}$. We also define the Esscher transform via

$$
\left.\frac{\mathrm{dP}_{x}^{c}}{\mathrm{dP}_{x}}\right|_{\mathcal{F}_{t}}=\frac{\mathcal{E}_{t}(c)}{\mathcal{E}_{0}(c)}
$$

for any $c$ for which $\mathrm{E}^{c X_{1}}<\infty$, where $\varepsilon_{t}(c)=\exp \left\{c X_{t}-\psi(c) t\right\}$ is the exponential martingale under $\mathrm{P}_{x}$. It is easy to check that, under this change of measure, $X$ remains within the class of spectrally negative processes and the Laplace exponent of $X$ under $\mathrm{P}^{c}$ is given by

$$
\varphi_{c}(\theta)=\varphi(\theta+c)-\varphi(c) \text { for } \theta \geq-c .
$$


Similarly, by (2) applied to $\mathrm{P}^{c}$, the exponent of the descending ladder process equals

$$
\hat{\kappa}_{c}(0, \beta)=\frac{\varphi(\beta+c)-\varphi(c)}{\beta},
$$

since, owing to the positive drift of $X$, we have $\Phi(0)=0$.

For $q \geq 0$, there exists a function $W^{(q)}:[0, \infty) \rightarrow[0, \infty)$, called the $q$-scale function, which is continuous and increasing with the Laplace transform

$$
\int_{0}^{\infty} \mathrm{e}^{-\theta y} W^{(q)}(y) \mathrm{d} y=(\varphi(\theta)-q)^{-1}, \quad \theta>\Phi(q) .
$$

We define $W^{(0)}(y)=W(y)$. The domain of $W^{(q)}$ is extended to the entire real axis by setting $W^{(q)}(y)=0$ for $y<0$. For each $y \geq 0$, the function $q \rightarrow W^{(q)}(y)$ may be analytically extended to $q \in \mathbb{C}$. Moreover, let

$$
Z^{(q)}(y)=1+q \int_{0}^{y} W^{(q)}(z) \mathrm{d} z .
$$

It is known that

$$
\begin{aligned}
& \mathrm{E}_{x}\left(\mathrm{e}^{-\theta \tau_{y}^{+}}, \tau_{y}^{+}<\infty\right)=\mathrm{e}^{-\Phi(\theta)(y-x)} \\
& \mathrm{E}_{x}\left(\mathrm{e}^{-q \tau_{0}^{-}}, \tau_{0}^{-}<\infty\right)=Z^{(q)}(x)-\frac{q}{\Phi(q)} W^{(q)}(x), \\
& \mathrm{E}_{x}\left(\mathrm{e}^{v X_{\tau_{0}^{-}}}, \tau_{0}^{-}<\infty\right)=\mathrm{e}^{v x}\left(Z_{v}^{(-\varphi(v))}(x)+\frac{\varphi(v)}{\Phi(-\varphi(v))} W_{v}^{(-\varphi(v))}(x)\right),
\end{aligned}
$$

where $W_{v}^{(-\varphi(v))}$ and $Z_{v}^{(-\varphi(v))}$ are scale functions calculated with respect to the measure $\mathrm{P}^{v}$. We understand $\theta / \Phi(\theta)$ in the limiting sense for $\theta=0$, so that

$$
\mathrm{P}_{x}\left(\tau_{0}^{-}<\infty\right)= \begin{cases}1-\varphi^{\prime}(0) W(x) & \text { if } \varphi^{\prime}(0+)>0, \\ 1 & \text { if } \varphi^{\prime}(0+) \leq 0 .\end{cases}
$$

For details, see [17] or [18, Remark 3]. If $\mathrm{P}_{x}\left(X_{\tau_{0}^{-}}=0, \tau_{0}^{-}<\infty\right)>0$ then we say that $X$ creeps below level 0 . This is possible only when the descending ladder process has strictly positive drift $d$. In this case the renewal function $\hat{U}$ has a strictly positive and continuous density $\hat{u}$ on $(0, \infty)$ satisfying

$$
\mathrm{P}_{x}\left(X_{\tau_{0}^{-}}=0, \tau_{0}^{-}<\infty\right)=d \hat{u}(x),
$$

where $\hat{u}(0+)=1 / d$; see [17, Theorem 5.9 and Problem 5.5].

\section{Main representation}

The main representation is given in the next theorem.

Theorem 1. The Parisian ruin probability for a spectrally negative Lévy risk process is given by

$$
\begin{aligned}
\mathrm{P}_{x}\left(\tau^{\zeta}<\infty\right)= & \mathrm{P}_{x}\left(\tau_{0}^{-}<\infty\right) \mathrm{P}\left(\tau^{\zeta}<\infty\right) \\
& +\left(1-\mathrm{P}\left(\tau^{\zeta}<\infty\right)\right) \int_{0}^{\infty} \mathrm{P}\left(\tau_{z}^{+}>\zeta\right) \mathrm{P}_{x}\left(\tau_{0}^{-}<\infty,-X_{\tau_{0}^{-}} \in \mathrm{d} z\right)
\end{aligned}
$$


and

$$
\begin{gathered}
\mathrm{P}_{x}\left(\tau_{0}^{-}<\infty\right)=\hat{\kappa}(0,0) \hat{U}(x, \infty)=1-\varphi^{\prime}(0+) W(x), \\
\int_{0}^{\infty} \mathrm{e}^{-\theta s} \mathrm{~d} s \int_{0}^{\infty} \mathrm{P}\left(\tau_{z}^{+}>s\right) \mathrm{P}_{x}\left(\tau_{0}^{-}<\infty,-X_{\tau_{0}^{-}} \in \mathrm{d} z\right) \\
=\frac{\hat{\kappa}(0,0) \hat{U}(x, \infty)}{\theta}-\frac{\hat{\kappa}(0,0)}{\Phi(\theta)} \mathrm{e}^{\Phi(\theta) x} \int_{x}^{\infty} \mathrm{e}^{-\Phi(\theta) y} \hat{U}(\mathrm{~d} y) \\
=\frac{1-\varphi^{\prime}(0+) W(x)}{\theta}-\frac{1}{\theta} \mathrm{e}^{\Phi(\theta) x}\left(Z_{\Phi(\theta)}^{(-\theta)}(x)+\frac{\theta}{\Phi(-\theta)} W_{\Phi(\theta)}^{(-\theta)}(x)\right) .
\end{gathered}
$$

Proof. On the event $\left\{\tau^{\zeta}=\infty\right\}$ we decompose the possible trajectory that goes below 0 into two parts as follows. The first part starts at the undershoot of 0 of size, say, $-z<0$, visiting 0 in a continuous way, because of the spectral negativity of $X$, in a shorter period than $\zeta$. The second part starts at 0 after this excursion below 0 . Using the strong Markov property, we obtain

$$
\begin{aligned}
\mathrm{P}_{x}\left(\tau^{\zeta}=\infty\right)= & \mathrm{P}_{x}\left(\tau_{0}^{-}=\infty\right) \\
& +\mathrm{P}\left(\tau^{\zeta}=\infty\right) \int_{0}^{\infty} \mathrm{P}\left(\tau_{z}^{+} \leq \zeta\right) \mathrm{P}_{x}\left(\tau_{0}^{-}<\infty,-X_{\tau_{0}^{-}} \in \mathrm{d} z\right) .
\end{aligned}
$$

This justifies (10). Moreover, the identity

$$
\mathrm{P}_{x}\left(\tau_{0}^{-}<\infty\right)=\mathrm{P}\left(\hat{\tau}_{x}^{+}<\infty\right)
$$

and the observation that, for $x>0$,

$$
\mathrm{P}\left(\hat{\tau}_{x}^{+}<\infty\right)=\hat{\kappa}(0,0) \hat{U}(x, \infty)
$$

completes the proof of (11) in view of (9) (for (14), see [17, p. 187]). Finally, note that, from (7),

$$
\int_{0}^{\infty} \mathrm{e}^{-\theta s} \mathrm{P}\left(\tau_{z}^{+}>s\right) \mathrm{d} s=\frac{1}{\theta}\left(1-\mathrm{E}\left(\mathrm{e}^{-\theta \tau_{z}^{+}}, \tau_{z}^{+}<\infty\right)\right)=\frac{1}{\theta}\left(1-\mathrm{e}^{-\Phi(\theta) z}\right)
$$

Furthermore, in the case of downward creeping, the distribution $\mathrm{P}_{x}\left(\tau_{0}^{-}<\infty,-X_{\tau_{0}^{-}} \in \mathrm{d} z\right)$ might have an atom at 0 , but it does not have an influence on the right-hand side of (13):

$$
\begin{aligned}
\int_{0}^{\infty} & \mathrm{e}^{-\theta s} \mathrm{~d} s \int_{0}^{\infty} \mathrm{P}\left(\tau_{z}^{+}>s\right) \mathrm{P}_{x}\left(\tau_{0}^{-}<\infty,-X_{\tau_{0}^{-}} \in \mathrm{d} z\right) \\
& =\frac{1}{\theta} \int_{0}^{\infty}\left(1-\mathrm{e}^{-\Phi(\theta) z}\right) \mathrm{P}_{x}\left(\tau_{0}^{-}<\infty,-X_{\tau_{0}^{-}} \in \mathrm{d} z\right) \\
& =\frac{\mathrm{P}_{x}\left(\tau_{0}^{-}<\infty\right)}{\theta}-\frac{1}{\theta} \mathrm{E}_{x}\left(\mathrm{e}^{\Phi(\theta) X_{\tau_{0}^{-}}}, \tau_{0}^{-}<\infty\right)
\end{aligned}
$$

Identity (8) completes proof of (13). Equation (12) follows from the equality

$$
\mathrm{P}_{x}\left(-X_{\tau_{0}^{-}} \in \mathrm{d} z, \tau_{0}^{-}<\infty\right)=\mathrm{P}\left(\hat{X}_{\hat{\tau}_{x}^{+}}-x \in \mathrm{d} z, \hat{\tau}_{x}^{+}<\infty\right)
$$

and [17, Problem 5.5]. 
Remark 1. Note that $\mathrm{e}^{-\Phi(\theta) x} W(x)=W_{\Phi(\theta)}^{(-\theta)}(x)$ and $Z_{\Phi(\theta)}^{(-\theta)}(x)=1-\theta \int_{0}^{x} \mathrm{e}^{-\Phi(\theta) y} W(y) \mathrm{d} y$. Moreover,

$$
\int_{0}^{\infty} \mathrm{e}^{-\beta x} W_{\Phi(\theta)}^{(-\theta)}(x) \mathrm{d} x=\varphi(\beta+\Phi(\theta))^{-1}, \quad Z_{\Phi(\theta)}^{(-\theta)}(x)=1-\theta \int_{0}^{x} W_{\Phi(\theta)}^{(-\theta)}(y) \mathrm{d} y .
$$

Remark 2. Now consider the particular case of the spectrally negative Lévy process

$$
X_{t}=x+p t-S_{t}+\sigma B_{t},
$$

where $p>0, \sigma \geq 0, S_{t}$ is a subordinator of bounded variation with Lévy measure $\Pi_{\hat{X}}$, and $B_{t}$ is a Brownian motion independent of $S_{t}$. Moreover, let

$$
v_{0}=-\int_{-\infty}^{0} z \Pi_{X}(\mathrm{~d} z)=\int_{0}^{\infty} \bar{\Pi}_{\hat{X}}(z) \mathrm{d} z<\infty,
$$

where $\bar{\Pi}_{\hat{X}}(z)=\Pi_{\hat{X}}(z, \infty)$. Note that $\rho_{0}=\mathrm{E} S_{1} / p<1$ because $\mathrm{E} X_{1}>0$. We now recall the Pollaczek-Khintchine formula (see [14, Theorem 3.1]):

$$
\mathrm{P}_{x}\left(\tau_{0}^{-}<\infty\right)=\hat{\kappa}(0,0) \hat{U}(x, \infty)=1-\left(1-\rho_{0}\right) \sum_{n=0}^{\infty} \rho_{0}^{n}\left(K^{(n+1) *} * M^{n *}\right)(x) .
$$

Here

$$
M(\mathrm{~d} z)=\frac{1}{v_{0}} \bar{\Pi}_{\hat{X}}(z) \mathrm{d} z
$$

When $\sigma>0$,

$$
\int_{0}^{\infty} \mathrm{e}^{-\theta z} K(\mathrm{~d} z)=\frac{p \theta}{p \theta+\theta^{2} \sigma^{2} / 2}
$$

hence, $K(\mathrm{~d} z)=\left(2 p / \sigma^{2}\right) \mathrm{e}^{-(2 p z) / \sigma^{2}} \mathrm{~d} z$.

The $\sigma=0$ case. If there is no Gaussian component, that is, $X_{t}$ is a drift process minus the subordinator of bounded variation, then $K(\mathrm{~d} z)=\delta_{0}(\mathrm{~d} z)$. Equation (18) then allows us to find a more explicit expression for $\int_{0}^{\infty} \mathrm{e}^{-\theta s} \mathrm{~d} s \int_{0}^{\infty} \mathrm{P}\left(\tau_{z}^{+}>s\right) \mathrm{P}_{x}\left(\tau_{0}^{-}<\infty,-X_{\tau_{0}^{-}} \in \mathrm{d} z\right)$ using (11), (12), and (19). Moreover,

$$
\rho=\mathrm{P}\left(\tau_{0}^{-}<\infty\right)=1-\frac{\mathrm{E} X(1)}{p}=\frac{\mathrm{E} S_{1}}{p}=\rho_{0} .
$$

Remark 3. From the proof of Theorem 1, it is transparent that the process that evolves below 0 could be different (have a different Lévy triple) from that which is above 0 . This gives the additional possibility of modeling the costs of debt that an insurance company are liable for during the so-called red period. To simplify the exposition, we omit this possibility.

To identify the ruin probability $\mathrm{P}_{x}\left(\tau^{\zeta}<\infty\right)$, we need to find the constant $\mathrm{P}\left(\tau^{\zeta}<\infty\right)$, which is given in the next theorem.

Denote by $p^{+}(s)=\mathrm{P}_{\varepsilon}\left(\tau_{0}^{-}<s\right)$ the probability that the excursion above 0 is shorter than $s$, and let

$$
p(s, t)=\int_{0}^{\infty} \mathrm{P}\left(\tau_{z+\varepsilon}^{+} \leq t\right) \mathrm{P}_{\varepsilon}\left(\tau_{0}^{-}<s,-X_{\tau_{0}^{-}} \in \mathrm{d} z\right)+\mathrm{P}\left(\tau_{\varepsilon}^{+} \leq t\right) \mathrm{P}_{\varepsilon}\left(\tau_{0}^{-}<s, X_{\tau_{0}^{-}}=0\right)
$$


be the probability that the upper excursion above 0 is shorter than $s$ and the first consecutive excursion below 0 , which is shifted downward by $-\varepsilon$, is shorter then $t$. Note that $p^{+}(s)=$ $p(s, \infty)$. If the process $X$ is of bounded variation then it can be expressed in the form

$$
X_{t}=x+p t-S_{t},
$$

where $S_{t}$ is a strict subordinator without drift.

Theorem 2. (i) If $X$ is a process of bounded variation then

$$
\mathrm{P}\left(\tau^{\zeta}<\infty\right)=\frac{\int_{0}^{\infty} \mathrm{P}\left(\tau_{z}^{+}>\zeta\right) \mathrm{P}\left(\tau_{0}^{-}<\infty,-X_{\tau_{0}^{-}} \in \mathrm{d} z\right)}{1-\rho+\int_{0}^{\infty} \mathrm{P}\left(\tau_{z}^{+}>\zeta\right) \mathrm{P}\left(\tau_{0}^{-}<\infty,-X_{\tau_{0}^{-}} \in \mathrm{d} z\right)},
$$

where

$$
\begin{aligned}
\int_{0}^{\infty} & \mathrm{e}^{-\theta s} \mathrm{~d} s \int_{0}^{\infty} \mathrm{P}\left(\tau_{z}^{+}>s\right) \mathrm{P}\left(\tau_{0}^{-}<\infty,-X_{\tau_{0}^{-}} \in \mathrm{d} z\right) \\
& =\frac{1}{\theta p} \int_{0}^{\infty}\left(1-\mathrm{e}^{-\Phi(\theta) z}\right) \bar{\Pi}_{\hat{X}}(z) \mathrm{d} z \\
& =\frac{1}{p}\left(\frac{1}{\Phi(\theta)}-\frac{\varphi^{\prime}(0+)}{\theta}\right)
\end{aligned}
$$

with $p$ as defined in (21).

(ii) If $X$ is a process of unbounded variation then

$$
\mathrm{P}\left(\tau^{\zeta}<\infty\right)=\lim _{b \rightarrow \infty} \lim _{\varepsilon \downarrow 0} \frac{p^{+}(b)-p(b, \zeta)}{1-p(b, \zeta)} .
$$

Proof. If $X$ is a process of bounded variation then, by [3, Corollary VII.5], 0 is irregular for $(-\infty, 0)$. Since, by the drift assumption, we excluded the case of a compound Poisson process $X, 0$ is regular for $(0, \infty)$. Now (22) follows from Theorem 1 by taking $x=0$, and using identity (20) and [17, p. 105 and Corollary 7.5] (see also [14, Corollary 4.5]). Equation (23) follows from the representation of the Laplace exponent $\varphi(\theta)=p \theta-\int_{0}^{\infty}\left(1-\mathrm{e}^{-\theta z}\right) \Pi_{\hat{X}}(\mathrm{~d} z)$ and the identity $\theta=\varphi(\Phi(\theta))$.

To prove (ii), we adapt the ideas of the proofs of the main results of Dassios and Wu [8], [10].

Let $\delta_{0}^{ \pm}=0$. First we define the sequence of stopping times

$$
\begin{array}{ll}
\sigma_{n}^{+}=\inf \left\{t>\delta_{n}^{+}: X_{t} \leq-\varepsilon\right\}, & \delta_{n+1}^{+}=\inf \left\{t>\sigma_{n}^{+}: X_{t}=0\right\}, \\
\sigma_{n}^{-}=\inf \left\{t>\delta_{n}^{-}: X_{t}=\varepsilon\right\}, & \delta_{n+1}^{-}=\inf \left\{t>\sigma_{n}^{-}: X_{t} \leq 0\right\},
\end{array}
$$

and the processes

$$
X_{t}^{ \pm}= \begin{cases}X_{t} \pm \varepsilon & \text { if } \delta_{n}^{ \pm} \leq t<\sigma_{n}^{ \pm} \\ X_{t} & \text { if } \sigma_{n}^{ \pm} \leq t<\delta_{n+1}^{ \pm}\end{cases}
$$

Moreover, let $b>0$ and

$$
\tau_{b}=\inf \left\{t>0: t-\sup \left\{s<t: X_{s} \leq 0\right\}>b, X_{t}>0\right\}
$$


with the convention that $\sup \left\{s<t: X_{s} \leq 0\right\}=0$ if $X_{s}>0$ for all $s \leq t$. We similarly define the stopping times $\tau_{b}^{ \pm}$and $\tau_{ \pm}^{\zeta}$ for $X^{ \pm}$. Observe that

$$
\mathrm{P}\left(\tau_{+}^{\zeta}<\tau_{b}^{+}\right) \leq \mathrm{P}\left(\tau^{\zeta}<\tau_{b}\right) \leq \mathrm{P}\left(\tau_{-}^{\zeta}<\tau_{b}^{-}\right)
$$

To find $\mathrm{P}\left(\tau_{+}^{\zeta}<\tau_{b}^{+}\right)$, we first decompose the path of the process $X^{+}$into excursions above and below 0 . Formally, the probability of event $A_{j}$, the first excursion below 0 of length greater than $\zeta$ is the $j$ th excursion and it happens before the first excursion above 0 longer than $b$, is

$$
\mathrm{P}\left(A_{j}\right)=p(b, \zeta)^{j-1}\left(p^{+}(b)-p(b, \zeta)\right) .
$$

Summing over $j=1,2, \ldots$ gives

$$
\mathrm{P}\left(\tau_{+}^{\zeta}<\tau_{b}^{+}\right)=\frac{p^{+}(b)-p(b, \zeta)}{1-p(b, \zeta)} .
$$

Similarly,

$$
\mathrm{P}\left(\tau_{-}^{\zeta}<\tau_{b}^{-}\right)=\mathrm{P}\left(\tau_{\varepsilon}^{+}>\zeta\right)+\mathrm{P}\left(\tau_{\varepsilon}^{+} \leq \zeta\right) \frac{p^{+}(b)-p(b, \zeta)}{1-p(b, \zeta)}
$$

Recall that the process of bounded variation $X$ was excluded from our considerations and, hence, 0 is regular for $(0, \infty)$ (see [17, Theorem 6.5, p. 142]). Straightforward consequences of this fact are that $\lim _{\varepsilon \downarrow 0} \mathrm{P}\left(\tau_{\varepsilon}^{+} \leq \zeta\right)=1$, and that $\mathrm{P}\left(\tau_{-}^{\zeta}<\tau_{b}^{-}\right)$and $\mathrm{P}\left(\tau_{+}^{\zeta}<\tau_{b}^{+}\right)$have the same limits as $\varepsilon \downarrow 0$. From (25) we then derive the assertion of the theorem.

Remark 4. The probability $1-p(s, t)$ could be identified using a double Laplace transform. Indeed, by [17, Exercise 6.7(i), p. 176] and (7),

$$
\begin{aligned}
\beta \omega \int_{0}^{\infty} \int_{0}^{\infty}(1-p(s, t)) \mathrm{e}^{-\beta t} \mathrm{e}^{-\omega s} \mathrm{~d} t \mathrm{~d} s & =1-\mathrm{e}^{-\Phi(\beta) \varepsilon} \mathrm{E}_{\varepsilon}\left(\mathrm{e}^{-\Phi(\beta) X_{\tau_{0}^{-}}-\omega \tau_{0}^{-}}\right) \\
& =1-\frac{\mathrm{E}\left(\mathrm{e}^{\left.\Phi(\beta) \underline{X}_{\omega} \mathbf{1}_{\left\{-\underline{X}_{e_{\omega}}>\varepsilon\right\}}\right)}\right.}{\mathrm{Ee}^{\Phi(\beta) \underline{X}_{\omega}}},
\end{aligned}
$$

where $e_{\omega}$ is an independent of $X$ exponential random variable with intensity $\omega$. Moreover, from [18] we know that

$$
\mathrm{Ee}^{\Phi(\beta) \underline{X}_{e \omega}}=\frac{\omega(\Phi(\beta)-\Phi(\omega))}{\Phi(\omega)(\beta-\omega)}
$$

and that

$$
\mathrm{P}\left(-\underline{X}_{e_{\omega}} \in \mathrm{d} z\right)=\frac{\omega}{\Phi(\omega)} W^{(\omega)}(\mathrm{d} z)-\omega W^{(\omega)}(z) \mathrm{d} z .
$$

Note also that there always exists a function $n(\varepsilon)$ such that the limit

$$
m(\omega)=\lim _{\varepsilon \downarrow 0} \frac{\mathrm{P}\left(-\underline{X}_{e_{\omega}} \leq \varepsilon\right)}{n(\varepsilon)}
$$

is well defined and finite. We can set, for example, the denominator of (26) such that $m(w)=1$. For any $n(\varepsilon)$ satisfying (26), we have

$$
\lim _{\varepsilon \downarrow 0} \frac{1-p(s, t)}{n(\varepsilon)}=q(s, t),
$$


where

$$
\int_{0}^{\infty} \int_{0}^{\infty} \mathrm{e}^{-\beta t} \mathrm{e}^{-\omega s} q(s, t) \mathrm{d} t \mathrm{~d} s=\frac{m(\omega) \Phi(\omega)(\beta-\omega)}{\beta \omega^{2}(\Phi(\beta)-\Phi(\omega))}
$$

Then we also have

$$
\mathrm{P}\left(\tau^{\zeta}<\infty\right)=\lim _{b \rightarrow \infty} \frac{q(b, \zeta)-q(b, \infty)}{q(b, \zeta)} .
$$

\section{Cramér's estimate}

In this section we derive the exponential asymptotics of the Parisian ruin probability. Assume that Cramér's conditions are satisfied, that is, there exists a $\gamma>0$ satisfying

$$
\hat{\varphi}(\gamma)=\varphi(-\gamma)=0
$$

and $\hat{\varphi}(\theta)$ is finite in the neighborhood of $\gamma$. Then $\mathrm{Ee}^{-\gamma X_{1}}<\infty$ and we can define a new measure $\mathrm{P}^{-\gamma}$ via (4). Define $\hat{U}_{\gamma}(\mathrm{d} x)=\hat{U}_{\gamma}^{(0)}(\mathrm{d} x):=\mathrm{e}^{\gamma x} \hat{U}(\mathrm{~d} x)$ and

$$
\mu=\int_{0}^{\infty} x \hat{U}_{\gamma}^{(1)}(\mathrm{d} x)
$$

where $\hat{U}_{\gamma}^{(q)}(\mathrm{d} x)=\int_{0}^{\infty} \mathrm{e}^{-(q t+\gamma x)} \mathrm{P}\left(\hat{H}_{t} \in \mathrm{d} x\right) \mathrm{d} t$ for $q \geq 0$. Note from [4] that $\hat{U}_{\gamma}$ is a renewal function of the ladder height process calculated on $\mathrm{P}^{-\gamma}$. Moreover, from [4] we have

$$
\mu=\left.\frac{\partial \hat{\kappa}_{-\gamma}(0, \beta)}{\partial \beta}\right|_{\beta=0}=\left.\frac{\partial \hat{\kappa}(0, \beta)}{\partial \beta}\right|_{\beta=-\gamma} .
$$

Note also that the drift of $\hat{X}$ on $\mathrm{P}^{-\gamma}$ is positive, since, by (5), its Laplace exponent equals

$$
\hat{\varphi}_{-\gamma}(\theta)=\varphi(-\gamma-\theta),
$$

and, hence, $\hat{\varphi}_{-\gamma}^{\prime}(0+)=-\varphi^{\prime}(-\gamma-)>0$. Thus,

$$
\mathrm{P}^{-\gamma}\left(\hat{\tau}_{x}^{+}<\infty\right)=1
$$

Theorem 3. We assume that Cramér's conditions (28) hold. We also assume that the support of $\hat{\Pi}$ is not lattice when $\hat{\Pi}(\mathbb{R})<\infty$. We have

$$
\lim _{x \uparrow \infty} \mathrm{e}^{\gamma x} \mathrm{P}_{x}\left(\tau^{\zeta}<\infty\right)=\mathrm{P}\left(\tau^{\zeta}<\infty\right) \frac{\hat{\kappa}(0,0)}{\gamma \mu}+\left(1-\mathrm{P}\left(\tau^{\zeta}<\infty\right)\right) f^{(c)}(\zeta),
$$

where

$$
\int_{0}^{\infty} \mathrm{e}^{-\theta s} f^{(c)}(s) \mathrm{d} s=\frac{\hat{\kappa}(0,0)}{\gamma \mu \theta}-\frac{1}{(\gamma+\Phi(\theta))^{2} \mu}
$$

and $\mathrm{P}\left(\tau^{\zeta}<\infty\right)$ is given in Theorem 2. If $\mu=\infty$ then the left-hand side of (31) is understood to be 0 .

Proof. By (14) and the key renewal theorem, which states that $\hat{U}_{\gamma}(\mathrm{d} x)$ on $(0, \infty)$ converges weakly as a measure to $\mu^{-1} \mathrm{~d} x$ (see [4] and [17, p. 188]), we have

$$
\lim _{x \uparrow \infty} \mathrm{e}^{\gamma x} \mathrm{P}\left(\hat{\tau}_{x}^{+}<\infty\right)=\hat{\kappa}(0,0) \lim _{x \uparrow \infty} \mathrm{e}^{\gamma x} \hat{U}(x, \infty)=\hat{\kappa}(0,0) \lim _{x \uparrow \infty} \hat{U}_{\gamma}(x, \infty)=\frac{\hat{\kappa}(0,0)}{\gamma \mu} .
$$


Moreover, by (16) and (17),

$$
\begin{gathered}
\int_{0}^{\infty} \mathrm{e}^{-\theta s} \mathrm{~d} s \int_{0}^{\infty} \mathrm{P}\left(\tau_{z}^{+}>s\right) \mathrm{P}_{x}\left(\tau_{0}^{-}<\infty,-X_{\tau_{0}^{-}} \in \mathrm{d} z\right) \\
=\frac{\mathrm{P}\left(\hat{\tau}_{x}^{+}<\infty\right)}{\theta}-\frac{1}{\theta} \mathrm{E}\left[\mathrm{e}^{-\Phi(\theta)\left(\hat{X}_{\hat{\tau}_{x}^{+}}-x\right)}, \hat{\tau}_{x}^{+}<\infty\right],
\end{gathered}
$$

and, by (30), the optional stopping theorem, and [17, Problem 5.5], we have

$$
\begin{aligned}
\lim _{x \uparrow \infty} \mathrm{e}^{\gamma x} \mathrm{E}\left[\mathrm{e}^{-\Phi(\theta)\left(\hat{X}_{\hat{\tau}_{x}^{+}}-x\right)}, \hat{\tau}_{x}^{+}<\infty\right] \\
=\lim _{x \uparrow \infty} \mathrm{E}^{-\gamma}\left[\mathrm{e}^{-(\Phi(\theta)+\gamma)\left(\hat{X}_{\hat{\tau}_{x}^{+}}-x\right)}, \hat{\tau}_{x}^{+}<\infty\right] \\
=\lim _{x \uparrow \infty} \mathrm{E}^{-\gamma}\left[\mathrm{e}^{-(\Phi(\theta)+\gamma)\left(\hat{X}_{\hat{\tau}_{x}^{+}}-x\right)}\right] \\
=\hat{\kappa}_{-\gamma}(0, \Phi(\theta)+\gamma) \lim _{x \uparrow \infty} \int_{x}^{\infty} \mathrm{e}^{-(\Phi(\theta)+\gamma)(y-x)} \hat{U}_{\gamma}(\mathrm{d} y) \\
=\hat{\kappa}_{-\gamma}(0, \Phi(\theta)+\gamma) \lim _{x \uparrow \infty} \int_{0}^{\infty} \mathrm{e}^{-(\Phi(\theta)+\gamma) y} \hat{U}_{\gamma}(x+\mathrm{d} y) \\
=\frac{\varphi(\Phi(\theta))}{(\gamma+\Phi(\theta))^{2} \mu} \\
=\frac{\theta}{(\gamma+\Phi(\theta))^{2} \mu} .
\end{aligned}
$$

Taking $f^{(c)}(s)=\int_{0}^{\infty} \mathrm{P}\left(\tau_{z}^{+}>s\right) \mathrm{P}_{x}\left(\tau_{0}^{-}<\infty,-X_{\tau_{0}^{-}} \in \mathrm{d} z\right)$ in the above observations completes the proof in view of Theorem 1 .

\section{Convolution-equivalent case}

In this section we deal with a Lévy process $X$ for which $\Pi_{\hat{X}}$, the Lévy measure of its dual process, belongs to the class $f^{(\alpha)}$. This class is defined as follows (see [16] for the analysis of the classical ruin probability).

Definition 1. $\left(\right.$ Class $\mathcal{L}^{(\alpha)}$.) For a parameter $\alpha \geq 0$, we say that the measure $G$ on $[0, \infty)$ with tail $\bar{G}(x)=G(x, \infty)$ belongs to the class $\mathcal{L}^{(\alpha)}$ if

(i) $\bar{G}(x)>0$ for each $x \geq 0$,

(ii) $\lim _{u \rightarrow \infty} \bar{G}(u-x) / \bar{G}(u)=\mathrm{e}^{\alpha x}$ for each $x \in \mathbb{R}$, and $G$ is nonlattice,

(iii) $\lim _{n \rightarrow \infty} \bar{G}(n-1) / \bar{G}(n)=\mathrm{e}^{\alpha}$ if $G$ is lattice (then assumed of span 1).

Definition 2. (Class $\AA^{(\alpha)}$.) We say that $G$ belongs to the class $\wp^{(\alpha)}$ if

(i) $G \in \mathcal{L}^{(\alpha)}$,

(ii) for some $M_{0}<\infty$, we have

$$
\lim _{u \rightarrow \infty} \frac{\overline{G^{* 2}}(u)}{\bar{G}(u)}=2 M_{0},
$$

where $\overline{G^{* 2}}(u)=1-G^{* 2}(u)$ and '*' denotes the convolution. 
For all $a \in \mathbb{R}$ such that the following integral is finite, we define the moment generating function $\delta$ such that

$$
\delta_{a}(G)=\int_{0}^{\infty} \mathrm{e}^{a u} G(\mathrm{~d} u)
$$

Similar definitions could be given for a tail of any other measure.

Recall that $\hat{X}=-X$ is a spectrally positive Lévy process. Throughout this section, we assume that, for $\hat{X}$ and some fixed $\alpha \geq 0$, we have

(C1) if $\alpha>0$,

$$
\Pi_{\hat{X}} \in s^{(\alpha)}
$$

and

$$
\int_{0}^{x} \bar{\Pi}_{\hat{X}}(y) \mathrm{d} y \in 8^{(0)}
$$

(C2) if $\alpha>0$,

$$
\hat{\varphi}(\alpha)<0,
$$

(C3) for $q=\lim _{\beta \downarrow 0}-\hat{\varphi}(-\beta) / \kappa(0,-\beta)$,

$$
\mathrm{e}^{-q} \delta_{\alpha}(\hat{H})<1
$$

By $\delta_{\alpha}(\hat{H})$ we denote the moment generating function (32) of the distribution function of $\hat{H}_{1}$. Condition $(\mathrm{C} 1)$ gives

$$
\bar{\Pi}_{\hat{H}} \in \delta^{(\alpha)} .
$$

Condition (C3) has a force when $\alpha>0$; by the drift assumption, for $\alpha=0$, this condition is automatically satisfied.

We write $f(x) \sim g(x)$ if and only if $\lim _{x \rightarrow \infty} f(x) / g(x)=1$.

Theorem 4. Under assumptions (33)-(36), the asymptotic Parisian ruin probability equals

$$
\mathrm{P}_{x}\left(\tau^{\zeta}<\infty\right) \sim \operatorname{E} X_{1}\left(\frac{\alpha}{\hat{\varphi}(\alpha)}\right)^{2}\left(\mathrm{P}\left(\tau^{\zeta}<\infty\right)+\left(1-\mathrm{P}\left(\tau^{\zeta}<\infty\right)\right) f^{(e)}(\zeta)\right) \int_{x}^{\infty} \bar{\Pi}_{\hat{X}}(y) \mathrm{d} y,
$$

where

$$
\int_{0}^{\infty} \mathrm{e}^{-\theta s} f^{(e)}(s) \mathrm{d} s=\frac{1}{\theta} \int_{0}^{\infty}\left(1-\mathrm{e}^{-\Phi(\theta) z}\right) B(z) \mathrm{d} z
$$

and

$$
B(z)=\frac{\mathrm{e}^{-\alpha z}}{\operatorname{E} X_{1}}\left(-\hat{\varphi}(\alpha)+\alpha \int_{z}^{\infty} \mathrm{e}^{\alpha y} \bar{\Pi}_{\hat{X}}(y) \mathrm{d} y\right) .
$$

For $\alpha=0$, the term $-\hat{\varphi}(\alpha) / \alpha$ is understood in the limiting sense and equals $-\hat{\varphi}^{\prime}(0+)=\mathrm{E} X_{1}$.

Proof. From [16, Theorem 6.2] we have

$$
\mathrm{P}\left(\hat{\tau}_{x}^{+}<\infty\right)=\hat{\kappa}(0,0) \hat{U}(x, \infty) \sim \mathrm{E} X_{1}\left(\frac{\alpha}{\hat{\varphi}(\alpha)}\right)^{2} \int_{x}^{\infty} \bar{\Pi}_{\hat{X}}(y) \mathrm{d} y
$$

and

as $x \uparrow \infty$, where

$$
\mathrm{P}\left(\hat{X}_{\hat{\tau}_{x}^{+}}-x \in \mathrm{d} z \mid \hat{\tau}_{x}^{+}<\infty\right) \stackrel{\mathrm{D}}{=} B(z) \mathrm{d} z
$$

$$
B(z)=-\frac{\mathrm{d}}{\mathrm{d} z} \frac{\mathrm{e}^{-\alpha z}}{\mathrm{E} X_{1}}\left(-\frac{\hat{\varphi}(\alpha)}{\alpha}+\int_{z}^{\infty}\left(\mathrm{e}^{\alpha y}-\mathrm{e}^{\alpha z}\right) \bar{\Pi}_{\hat{X}}(y) \mathrm{d} y\right) .
$$

Equations (15) and (17), dominated convergence theorem and Theorem 1 complete the proof. 


\section{Examples}

\subsection{General classic risk process (1)}

For the process (1), we have $\Pi_{\hat{X}}(\mathrm{~d} z)=\lambda F(\mathrm{~d} z)$ and $\varphi(\theta)=p \theta-\lambda+\lambda \int_{0}^{\infty} \mathrm{e}^{-\theta z} F(\mathrm{~d} z)$. By [14, Corollary 4.5] and [17, Corollary 4.12, p. 105],

$$
\mathrm{P}\left(\tau_{0}^{-}<\infty,-X_{\tau_{0}^{-}} \in \mathrm{d} z\right)=\frac{\lambda}{p} \bar{F}(z) \mathrm{d} z
$$

and, hence,

$$
\begin{gathered}
\int_{0}^{\infty} \mathrm{e}^{-\theta s} \mathrm{~d} s \int_{0}^{\infty} \mathrm{P}\left(\tau_{z}^{+}>s\right) \mathrm{P}\left(\tau_{0}^{-}<\infty,-X_{\tau_{0}^{-}} \in \mathrm{d} z\right) \\
=\frac{\lambda}{\theta p} \int_{0}^{\infty}\left(1-\mathrm{e}^{-\Phi(\theta) z}\right) \bar{F}(z) \mathrm{d} z
\end{gathered}
$$

The probability $\mathrm{P}\left(\tau^{\zeta}<\infty\right)$ could be found using Theorem 2 with (38) and

$$
\rho=\mathrm{P}\left(\tau_{0}^{-}<\infty\right)=\frac{\lambda \nu}{p},
$$

where $v=\int_{0}^{\infty} y F(\mathrm{~d} y)$. Theorem 1 then gives the Parisian ruin probability $\mathrm{P}_{x}\left(\tau^{\zeta}<\infty\right)$ for all $x \geq 0$.

To find the Cramér asymptotics, note that $\Phi(\theta)$ and $\gamma$ solve the equations

$$
\int_{0}^{\infty} \mathrm{e}^{-\Phi(\theta) z} F(\mathrm{~d} z)=\frac{\lambda-p \Phi(\theta)+\theta}{\lambda}, \quad \int_{0}^{\infty} \mathrm{e}^{\gamma z} F(\mathrm{~d} z)=\frac{\lambda+p \gamma}{\lambda} .
$$

Moreover, from (3) we have

$$
\hat{\kappa}(0,0)=\varphi^{\prime}(0+)=p-\lambda \nu .
$$

By (29), using the fact that $\varphi(-\gamma)=0$, we have

$$
\mu=\lambda \int_{0}^{\infty} y \mathrm{e}^{\gamma y} \Pi_{\hat{X}}(y, \infty) \mathrm{d} y=\lambda \int_{0}^{\infty} y \mathrm{e}^{\gamma y} \bar{F}(y) \mathrm{d} y,
$$

and, hence,

$$
\lim _{x \uparrow \infty} \mathrm{e}^{\gamma x} \mathrm{P}_{x}\left(\tau^{\zeta}<\infty\right)=\mathrm{P}\left(\tau^{\zeta}<\infty\right) \frac{p-\lambda \nu}{\gamma \mu}+\left(1-\mathrm{P}\left(\tau^{\zeta}<\infty\right)\right) f^{(c)}(\zeta),
$$

where

$$
\int_{0}^{\infty} \mathrm{e}^{-\theta s} f^{(c)}(s) \mathrm{d} s=\frac{1}{\mu}\left(\frac{p-\lambda v}{\gamma \theta}-\frac{1}{(\gamma+\Phi(\theta))^{2}}\right) .
$$

If $F \in 8^{(\alpha)}$ for $\alpha \geq 0$ then $\mathrm{P}\left(\tau^{\zeta}<\infty\right)$ is as given in Theorem 4 .

\subsection{Classic risk process (1) with exponential jumps}

Corollary 1. Assume that $X_{t}$ is the Cramér-Lundberg risk process (1) with exponential claims $F(\mathrm{~d} z)=\xi \mathrm{e}^{-\xi z} \mathrm{~d} z$, where $\xi=1 / \nu$. Then, for $x \geq 0$,

$$
\mathrm{P}_{x}\left(\tau^{\zeta}<\infty\right)=\frac{\lambda}{p \xi} \mathrm{e}^{-(p \xi-\lambda) x / p}\left(\frac{p \xi D}{p \xi-\lambda(1-D)}\right),
$$


where

$$
D=1-\int_{0}^{\zeta} \sqrt{\frac{p \xi}{\lambda}} \mathrm{e}^{-(\lambda+p \xi) t} t^{-1} I_{1}(2 t \sqrt{p \lambda \xi}) \mathrm{d} t
$$

and $I_{1}(x)$ is the modified Bessel function of the first kind.

Proof. From (6) and (11) (see also [2, p. 63]), we have

$$
\hat{\kappa}(0,0) \hat{U}(x, \infty)=\frac{\lambda}{p \xi} \mathrm{e}^{-(p \xi-\lambda) x / p} .
$$

Moreover, by the lack-of-memory property of exponential distributions, the distribution of the undershoot of 0 is also exponential with intensity $\xi$. Hence,

$$
\int_{0}^{\infty} \mathrm{e}^{-\theta s} \mathrm{~d} s \int_{0}^{\infty} \mathrm{P}\left(\tau_{z}^{+}>s\right) \mathrm{P}_{x}\left(\tau_{0}^{-}<\infty,-X_{\tau_{0}^{-}} \in \mathrm{d} z\right)=\frac{\lambda}{p \xi} \mathrm{e}^{-(p \xi-\lambda) x / p} \frac{\Phi(\theta)}{\theta(\Phi(\theta)+\xi)}
$$

and, by (38),

$$
\int_{0}^{\infty} \mathrm{e}^{-\theta s} \mathrm{~d} s \int_{0}^{\infty} \mathrm{P}\left(\tau_{z}^{+}>s\right) \mathrm{P}\left(\tau_{0}^{-}<\infty,-X_{\tau_{0}^{-}} \in \mathrm{d} z\right)=\frac{\lambda}{p \xi} \frac{\Phi(\theta)}{\theta(\Phi(\theta)+\xi)} .
$$

Inverting the Laplace transforms (41) and (42) with respect to $\theta$ (see [12]) gives

$$
\int_{0}^{\infty} \mathrm{P}\left(\tau_{z}^{+}>\zeta\right) \mathrm{P}_{x}\left(\tau_{0}^{-}<\infty,-X_{\tau_{0}^{-}} \in \mathrm{d} z\right)=\frac{\lambda}{p \xi} \mathrm{e}^{-(p \xi-\lambda) x / p} D
$$

and

$$
\int_{0}^{\infty} \mathrm{P}\left(\tau_{z}^{+}>\zeta\right) \mathrm{P}\left(\tau_{0}^{-}<\infty,-X_{\tau_{0}^{-}} \in \mathrm{d} z\right)=\frac{\lambda}{p \xi} D .
$$

Furthermore, from Theorem 2 we have

$$
\mathrm{P}\left(\tau^{\zeta}<\infty\right)=\frac{\lambda D / p \xi}{(p \xi-\lambda) / p \xi+\lambda D / p \xi} .
$$

Representation (10) given in Theorem 1 and identities (40), (43), and (44) complete the proof of (39).

The Parisian probability in this case has already been identified in [8].

\subsection{Brownian motion with drift}

Corollary 2. Assume that

$$
X_{t}=x+\sigma B_{t}+p t,
$$

where $\sigma, p>0$ and $B_{t}$ is a standard Brownian motion. Then, for $x \geq 0$,

$$
\mathrm{P}_{x}\left(\tau^{\zeta}<\infty\right)=\mathrm{e}^{-\left(2 p \sigma^{-2}\right) x}\left(\Psi\left(\frac{p}{\sigma} \sqrt{\frac{\zeta}{2}}\right)-\frac{p}{\sigma} \sqrt{\frac{\zeta \pi}{2}}\right)\left(\Psi\left(\frac{p}{\sigma} \sqrt{\frac{\zeta}{2}}\right)+\frac{p}{\sigma} \sqrt{\frac{\zeta \pi}{2}}\right)^{-1},
$$

where

$$
\Psi(x)=2 \sqrt{\pi} x \mathcal{N}(\sqrt{2} x)-\sqrt{\pi} x+\mathrm{e}^{-x^{2}}
$$

and $\mathcal{N}(\cdot)$ is a cumulative distribution function for the standard normal distribution. 
Proof. Note that

$$
\int_{0}^{\infty} \mathrm{P}\left(\tau_{z}^{+}>\zeta\right) \mathrm{P}_{x}\left(\tau_{0}^{-}<\infty,-X_{\tau_{0}^{-}} \in \mathrm{d} z\right)=0 .
$$

Moreover, using

$$
\varphi(\beta)=p \beta+\frac{\sigma^{2} \beta^{2}}{2}
$$

and (6), we have

$$
W(x)=\frac{1}{p}\left(1-\mathrm{e}^{-\left(2 p \sigma^{-2}\right) x}\right)
$$

and, hence, from (11),

$$
\mathrm{P}_{x}\left(\tau_{0}^{-}<\infty\right)=\mathrm{e}^{-\left(2 p \sigma^{-2}\right) x} .
$$

The first passage probability $\tau_{\varepsilon}^{+}$has inverse Gaussian distribution and

$$
\mathrm{P}\left(\tau_{\varepsilon}^{+}<\zeta\right)=\mathcal{N}\left(\frac{p}{\sigma} \sqrt{\zeta}-\frac{\varepsilon}{\sigma \sqrt{\zeta}}\right)+\mathrm{e}^{2 p \varepsilon \sigma^{-2}} \mathcal{N}\left(-\frac{p}{\sigma} \sqrt{\zeta}-\frac{\varepsilon}{\sigma \sqrt{\zeta}}\right) .
$$

Similarly,

$$
\mathrm{P}_{\varepsilon}\left(\tau_{0}^{-}<b\right)=\mathrm{e}^{-2 p \varepsilon \sigma^{-2}} \mathcal{N}\left(\frac{p}{\sigma} \sqrt{b}-\frac{\varepsilon}{\sigma \sqrt{b}}\right)+\mathcal{N}\left(-\frac{p}{\sigma} \sqrt{b}-\frac{\varepsilon}{\sigma \sqrt{b}}\right) .
$$

From (47) and (48), using the continuity of Brownian paths, we have

$$
\begin{aligned}
\lim _{\varepsilon \downarrow 0} \frac{p^{+}(b)-p(b, \zeta)}{1-p(b, \zeta)} \\
\quad=\lim _{\varepsilon \downarrow 0} \frac{\mathrm{P}_{\varepsilon}\left(\tau_{0}^{-}<b\right) \mathrm{P}\left(\tau_{\varepsilon}^{+}>\zeta\right)}{1-\mathrm{P}_{\varepsilon}\left(\tau_{0}^{-}<b\right) \mathrm{P}\left(\tau_{\varepsilon}^{+} \leq \zeta\right)} \\
=\left(\sqrt{b} \Psi\left(-\frac{p}{\sigma} \sqrt{\frac{\zeta}{2}}\right)-\frac{p}{\sigma} \sqrt{\frac{b \zeta \pi}{2}}\right)\left(\sqrt{b} \Psi\left(-\frac{p}{\sigma} \sqrt{\frac{\zeta}{2}}\right)+\sqrt{\zeta} \Psi\left(\frac{p}{\sigma} \sqrt{\frac{b}{2}}\right)\right)^{-1} \\
=\left(\sqrt{b} \Psi\left(\frac{p}{\sigma} \sqrt{\frac{\zeta}{2}}\right)-\frac{p}{\sigma} \sqrt{\frac{b \zeta \pi}{2}}\right)\left(\sqrt{b} \Psi\left(\frac{p}{\sigma} \sqrt{\frac{\zeta}{2}}\right)+\sqrt{\zeta} \Psi\left(\frac{p}{\sigma} \sqrt{\frac{b}{2}}\right)\right)^{-1}
\end{aligned}
$$

where we used the fact that $\Psi(-x)=\Psi(x)$. Note also that

$$
\begin{aligned}
\lim _{b \rightarrow \infty} & \sqrt{\frac{\zeta}{b}} \Psi\left(\frac{p}{\sigma} \sqrt{\frac{b}{2}}\right) \\
& =\lim _{b \rightarrow \infty} \sqrt{\frac{\zeta}{b}}\left(2 \sqrt{\pi} \frac{p}{\sigma} \sqrt{\frac{b}{2}} \mathcal{N}\left(\frac{p}{\sigma} \sqrt{b}\right)-\sqrt{\pi} \frac{p}{\sigma} \sqrt{\frac{b}{2}}+\mathrm{e}^{-(p / \sigma)^{2} b / 2}\right) \\
& =\sqrt{\zeta} \frac{p}{\sigma} \sqrt{\frac{\pi}{2}} .
\end{aligned}
$$

Substituting (24) and (46) into (10) completes the proof of (45).

Probability (45) was also given in [8]. 


\subsection{General classic risk process perturbed by independent Brownian motion}

One of the main goals of this paper was to identify the Parisian ruin probability for more complex spectrally negative Lévy processes, typical examples of which we now analyze. We assume that

$$
X_{t}=x+p t-\sum_{i=1}^{N_{t}} U_{i}+\sigma B_{t},
$$

where $p, \sigma>0$, and $B_{t}$ is independent of the Poisson process $N_{t}$ with intensity $\lambda$ and claim arrival sequence $\left\{U_{i}\right\}_{i=1,2, \ldots}$. Then $\varphi(\theta)=p \theta-\lambda+\lambda \int_{0}^{\infty} \mathrm{e}^{-\theta z} F(\mathrm{~d} z)+\sigma^{2} \theta^{2} / 2$ and $\Phi(\theta)$ solves the equation

$$
\int_{0}^{\infty} \mathrm{e}^{-\Phi(\theta) z} F(\mathrm{~d} z)=\frac{1}{\lambda}\left(\lambda-p \Phi(\theta)-\frac{\sigma^{2}}{2} \Phi^{2}(\theta)+\theta\right) .
$$

The Parisian ruin probability $\mathrm{P}_{x}\left(\tau^{\zeta}<\infty\right)$ is as given in Theorem 1 and all terms appearing there are given in Remark 2, except for $\mathrm{P}\left(\tau^{\zeta}<\infty\right)$.

Using Theorem 2(ii) and Remark 4, we will find $\mathrm{P}\left(\tau^{\zeta}<\infty\right)$. Since $X$ has a Gaussian component, then $W^{(\omega)}(0+)=0$, and from the Tauberian theorem (see, e.g. [5, Theorem 1.7.1']), it follows that

$$
\lim _{\varepsilon \downarrow 0} \frac{W^{(\omega)}(\varepsilon)}{\varepsilon}=\frac{2}{\sigma^{2}} .
$$

Thus, $n(\varepsilon)=\varepsilon$ and

$$
m(\omega)=\frac{2 \omega}{\sigma^{2} \Phi(\omega)} .
$$

The Parisian ruin probability $\mathrm{P}\left(\tau^{\zeta}<\infty\right)$ is given in (27) for

$$
\int_{0}^{\infty} \int_{0}^{\infty} \mathrm{e}^{-\beta t} \mathrm{e}^{-\omega s} q(s, t) \mathrm{d} t \mathrm{~d} s=\frac{2(\beta-\omega)}{\sigma^{2} \beta \omega(\Phi(\beta)-\Phi(\omega))} .
$$

Cramér asymptotics $\mathrm{P}_{x}\left(\tau^{\zeta}<\infty\right)$ are as given in Theorem 3, where $\gamma$ solves the equation

$$
\int_{0}^{\infty} \mathrm{e}^{\gamma z} F(\mathrm{~d} z)=\frac{1}{\lambda}\left(\lambda+p \gamma-\frac{\sigma^{2} \gamma^{2}}{2}\right)
$$

and $\hat{\kappa}(0,0)=\varphi^{\prime}(0+)=p-\lambda \nu$. Furthermore,

$$
\mu=\lambda \int_{0}^{\infty} y \mathrm{e}^{\gamma y} \bar{F}(y) \mathrm{d} y+\sigma .
$$

Assume now that the claim size has an exponential distribution $F(\mathrm{~d} z)=\xi \mathrm{e}^{-\xi z} \mathrm{~d} z$. Then, by [22] (see also [11]),

$$
\mathrm{P}_{x}\left(\tau_{0}^{-}<\infty\right)=\hat{\kappa}(0,0) \hat{U}(x, \infty)=1+\sum_{i=1}^{2} c_{i} \mathrm{e}^{-\varrho_{i} x},
$$

where

$$
\begin{aligned}
& \varrho_{1}=\frac{\left(\xi \sigma^{2} / 2+p\right)-\sqrt{\left(\xi \sigma^{2} / 2+p\right)^{2}-2 \sigma^{2}(\xi p-\lambda)}}{\sigma^{2}}, \\
& \varrho_{2}=\frac{\left(\xi \sigma^{2} / 2+p\right)+\sqrt{\left(\xi \sigma^{2} / 2+p\right)^{2}-2 \sigma^{2}(\xi p-\lambda)}}{\sigma^{2}},
\end{aligned}
$$




$$
\begin{aligned}
c_{1} & =\frac{\sigma^{2} \varrho_{2}^{2}-2 p \varrho_{2}}{\sigma^{2}\left(\varrho_{2}^{2}-\varrho_{1}^{2}\right)-2 p\left(\varrho_{2}-\varrho_{1}\right)}, \\
c_{2} & =\frac{-\sigma^{2} \varrho_{1}^{2}+2 p \varrho_{1}}{\sigma^{2}\left(\varrho_{2}^{2}-\varrho_{1}^{2}\right)-2 p\left(\varrho_{2}-\varrho_{1}\right)} .
\end{aligned}
$$

We have

$$
\begin{aligned}
\int_{0}^{\infty} & \mathrm{e}^{-\theta s} \mathrm{~d} s \int_{0}^{\infty} \mathrm{P}\left(\tau_{z}^{+}>s\right) \mathrm{P}_{x}\left(\tau_{0}^{-}<\infty,-X_{\tau_{0}^{-}} \in \mathrm{d} z\right) \\
& =\frac{1}{\theta} \mathrm{P}_{x}\left(\tau_{0}^{-}<\infty\right)\left(1-\xi \int_{0}^{\infty} \mathrm{e}^{-(\Phi(\theta)+\xi) s} \mathrm{~d} s\right) \\
& =\frac{1}{\theta} \mathrm{P}_{x}\left(\tau_{0}^{-}<\infty\right)\left(1-\frac{\xi}{\Phi(\theta)+\xi}\right)
\end{aligned}
$$

where $\Phi(\theta)$ solves the equation

$$
-\frac{\sigma^{2}}{2} \Phi^{3}(\theta)-\left(\xi \frac{\sigma^{2}}{2}+p\right) \Phi^{2}(\theta)+(\lambda+\theta-p \xi) \Phi(\theta)+\xi \theta=0
$$

and by inverting this Laplace transform we can identify $\int_{0}^{\infty} \mathrm{P}\left(\tau_{z}^{+}>\zeta\right) \mathrm{P}_{x}\left(\tau_{0}^{-}<\infty,-X_{\tau_{0}^{-}} \in\right.$ $\mathrm{d} z)$.

We now obtain

$$
\mathrm{P}\left(\tau^{\zeta}<\infty\right)=\lim _{b \rightarrow \infty} \lim _{\varepsilon \downarrow 0} \frac{(1-p(b, \zeta))-\mathrm{P}_{\varepsilon}\left(\tau_{0}^{-}>b\right)}{1-p(b, \zeta)}
$$

for the exponential claim size with intensity $\xi$. Note that, by (51), the Laplace transform $(1 / \theta)(1-\xi /(\Phi(\theta)+\xi))$ is then the only single Laplace transform that must be inverted at the point $s=\zeta$ to derive the Parisian ruin probability $\mathrm{P}_{x}\left(\tau^{\zeta}<\infty\right)$ (compare with (50), where the double Laplace transform must be inverted).

Recall that

$$
\begin{aligned}
p(b, \zeta)= & \left(\mathrm{P}_{\varepsilon}\left(\tau_{0}^{-}<b\right)-\mathrm{P}_{\varepsilon}\left(\tau_{0}^{-}<b, X_{\tau_{0}^{-}}=0\right)\right) \xi \int_{0}^{\infty} \mathrm{P}\left(\tau_{z+\varepsilon}^{+} \leq \zeta\right) \mathrm{e}^{-\xi z} \mathrm{~d} z \\
& +\mathrm{P}\left(\tau_{\varepsilon}^{+} \leq \zeta\right) \mathrm{P}_{\varepsilon}\left(\tau_{0}^{-}<b, X_{\tau_{0}^{-}}=0\right),
\end{aligned}
$$

and note that

$$
\begin{aligned}
\mathrm{P}_{\varepsilon}\left(\tau_{0}^{-}<b, X_{\tau_{0}^{-}}=0\right)= & \mathrm{P}_{\varepsilon}\left(\tau_{0}^{-}<\infty, X_{\tau_{0}^{-}}=0\right) \\
& -\int_{0}^{\infty} \mathrm{P}_{z}\left(\tau_{0}^{-}<\infty, X_{\tau_{0}^{-}}=0\right) \mathrm{P}_{\varepsilon}\left(\tau_{0}^{-} \geq b, X_{b} \in \mathrm{d} z\right) .
\end{aligned}
$$

Thus, from (53) and ([17, Theorem 5.9, p. 122], we obtain

$$
\lim _{\varepsilon \downarrow 0} \frac{\mathrm{P}_{\varepsilon}\left(\tau_{0}^{-}<b\right)-\mathrm{P}_{\varepsilon}\left(\tau_{0}^{-}<b, X_{\tau_{0}^{-}}=0\right)}{\varepsilon}=-d \hat{u}^{\prime}(0+)+d \int_{0}^{\infty} \hat{u}(z) g_{1}(b, \mathrm{~d} z)-g_{3}(b),
$$

where

$$
g_{1}(b, \mathrm{~d} z)=\lim _{\varepsilon \downarrow 0} \frac{\mathrm{P}_{\varepsilon}\left(\tau_{0}^{-} \geq b, X_{b} \in \mathrm{d} z\right)}{\varepsilon}
$$


and

$$
g_{3}(b)=\lim _{\varepsilon \downarrow 0} \frac{\mathrm{P}_{\varepsilon}\left(\tau_{0}^{-}>b\right)}{\varepsilon} .
$$

Then from (52) we have

$$
\mathrm{P}\left(\tau^{\zeta}<\infty\right)=\frac{g_{2}+g_{4}(\infty)-g_{3}(\infty)}{g_{2}+g_{4}(\infty)},
$$

where

$$
\begin{aligned}
g_{4}(b)= & d\left(-\hat{u}^{\prime}(0+)+\int_{0}^{\infty} \hat{u}(z) g_{1}(b, \mathrm{~d} z)\right)\left(1-\xi \int_{0}^{\infty} \mathrm{P}\left(\tau_{w}^{+} \leq \zeta\right) \mathrm{e}^{-\xi w} \mathrm{~d} w\right) \\
& +g_{3}(b) \xi \int_{0}^{\infty} \mathrm{P}\left(\tau_{w}^{+} \leq \zeta\right) \mathrm{e}^{-\xi w} \mathrm{~d} w
\end{aligned}
$$

$g_{2}=\lim _{\varepsilon \downarrow 0} \mathrm{P}\left(\tau_{\varepsilon}^{+}>\zeta\right) / \varepsilon$, and $g_{i}(\infty)=\lim _{b \rightarrow \infty} g_{i}(b), i=1,3,4$. The probability $\mathrm{P}\left(\tau_{w}^{+} \leq\right.$ $\zeta$ ) appearing in $g_{4}$ could be found using Kendall's identity, which states that if the spectrally negative $X_{s}$ has a density $m(s, w)$ at $w$ then $\tau_{w}^{+}$also has a density at $s$ and

$$
\frac{\mathrm{P}\left(\tau_{w}^{+} \in \mathrm{d} s\right)}{\mathrm{d} s}=\frac{w}{s} m(s, w),
$$

where in our case

$$
m(s, w)=\int_{x}^{\infty} \frac{\mathrm{d} y}{\sigma \sqrt{s}} \phi\left(\frac{y-p s}{\sigma \sqrt{s}}\right) \mathrm{e}^{-\lambda s-\zeta(y-x)} \frac{\sqrt{\lambda s \xi}}{\sqrt{y-x}} I_{1}(2 \sqrt{\lambda s \xi(y-x)}) .
$$

For Kendall's formula, see [3, Corollary VII.3], [6], and the references therein. We now find the functions $g_{i}$ for $i=1,2,3$.

To find $g_{1}(b, \mathrm{~d} z)$, note that the resolvent of the killed process $X_{t}$ when entering the negative half-line has the following resolvent density (see [17, p. 226]):

$$
r^{(q)}(x, y)=\mathrm{e}^{-\Phi(q) y} W^{(q)}(x)-W^{(q)}(x-y) .
$$

Hence, for $z>0$,

$$
\int_{0}^{\infty} \mathrm{e}^{-q b} \mathrm{P}_{\varepsilon}\left(\tau_{0}^{-} \geq b, X_{b} \in \mathrm{d} z\right) \mathrm{d} b=\left(\mathrm{e}^{-\Phi(q) z} W^{(q)}(\varepsilon)-W^{(q)}\left((\varepsilon-z)^{+}\right)\right) \mathrm{d} z,
$$

and then, by (49),

$$
\int_{0}^{\infty} \mathrm{e}^{-q b} g_{1}(b, \mathrm{~d} z) \mathrm{d} b=\frac{2}{\sigma^{2}} \mathrm{e}^{-\Phi(q) z} \mathrm{~d} z,
$$

since $W^{(q)}(0+)=0$ in our case. Moreover, from (7) we have

$$
\frac{g_{1}(b, \mathrm{~d} z)}{\mathrm{d} z}=\frac{\mathrm{P}\left(\tau_{z}^{+} \in \mathrm{d} b\right)}{\mathrm{d} b},
$$

where $\mathrm{P}\left(\tau_{z}^{+} \in \mathrm{d} b\right)$ is identified via (54).

The function $g_{3}(b)$ can be found by integrating $g_{1}(b, \mathrm{~d} z)$ using the dominated convergence theorem.

Finally, from (54) we have $g_{2}=\int_{b}^{\infty}(m(s, 0) / s) \mathrm{d} s$. 


\subsection{General classic risk process perturbed by an $\alpha$-stable motion}

We will now assume that

$$
X_{t}=x+p t-\sum_{i=1}^{N_{t}} U_{i}+Z_{t}
$$

where $p>0$, and $Z_{t}$ is a spectrally negative $\alpha$-stable motion with $\alpha \in(1,2)$, independent of the Poisson process $N_{t}$, with intensity $\lambda$ and claim arrival sequence $\left\{U_{i}\right\}_{i=1,2, \ldots}$. Note that, for $\alpha \in(1,2)$, process $Z$ is of unbounded variation. Moreover, process $X$ has no Gaussian component and it does not creep downward. Then $\varphi(\theta)=p \theta-\lambda+\lambda \int_{0}^{\infty} \mathrm{e}^{-\theta z} F(\mathrm{~d} z)+c \theta^{\alpha}$ for some $c>0$ and $\Phi(\theta)$ solves the equation

$$
\int_{0}^{\infty} \mathrm{e}^{-\Phi(\theta) z} F(\mathrm{~d} z)=\frac{\lambda-p \Phi(\theta)-c \Phi^{\alpha}(\theta)+\theta}{\lambda} .
$$

Then from [13] we have

$$
\mathrm{P}_{x}\left(\tau_{0}^{-}<\infty\right)=\hat{\kappa}(0,0) \hat{U}(x, \infty)=1-\left(1-\rho_{0}\right) \sum_{n=0}^{\infty} \rho_{0}^{n}\left(K^{(n+1) *} * M^{n *}\right)(x),
$$

where $\rho_{0}=\lambda \nu / p, M(\mathrm{~d} x)=(1 / v) \bar{F}(x) \mathrm{d} x$, and $\bar{K}(x)=\sum_{n=0}^{\infty}(-p)^{n} x^{(\alpha-1)} n / \Gamma(1+(\alpha-$ 1)n).

Finally, $\mathrm{P}_{x}\left(\tau^{\zeta}<\infty\right)$ can be found using the main representation given in Theorem 1, (12), and identity (56). Using Theorem 2(ii) and Remark 4, we can also identify $\mathrm{P}\left(\tau^{\zeta}<\infty\right)$, where similarly to the Brownian perturbation case we have $W^{(\omega)}(0+)=0$ and

$$
\lim _{\varepsilon \downarrow 0} \frac{W^{(\omega)}(\varepsilon)}{\varepsilon^{\alpha-1}}=\frac{1}{c \Gamma(\alpha)} .
$$

Thus, $n(\varepsilon)=\varepsilon^{\alpha-1}$ and

$$
m(\omega)=\frac{\omega}{c \Gamma(\alpha) \Phi(\omega)} .
$$

Note also that, for process (55), we have

$$
\Pi_{\hat{X}}(\mathrm{~d} y)=\frac{c}{y^{1+\alpha}} \mathrm{d} y+\lambda F(\mathrm{~d} y) .
$$

Assuming that $\bar{F}(x)=\mathrm{o}\left(x^{-\alpha}\right)$ for large $x$ we derive that $\bar{\Pi}_{\hat{X}} \in \delta^{(0)}$. From Theorem 4 we have

$$
\mathrm{P}_{x}\left(\tau^{\zeta}<\infty\right) \sim \frac{1}{\mathrm{E} X_{1}}\left(\mathrm{P}\left(\tau^{\zeta}<\infty\right)+\mathrm{P}\left(\tau^{\zeta}=\infty\right) f^{(e)}(\zeta)\right) \frac{c}{\alpha(\alpha-1)} x^{-\alpha+1}
$$

as $x \rightarrow \infty$, where the Laplace transform of $f^{(e)}(\cdot)$ is given in (37) for $\Pi_{\hat{X}}$ defined in (57).

\section{Acknowledgements}

This work was partially supported by the Ministry of Science and Higher Education of Poland, under grants N N201 394137 (2009-2011) and N N201 525638 (2010-2011). 


\section{References}

[1] Albrecher, H., Kortschak, D. and Zhou, X. (2010). Pricing of Parisian options for a jump-diffusion model with two-sided jumps. Submitted.

[2] Asmussen, S. (2000). Ruin Probabilities. World Scientific, River Edge, NJ.

[3] Bertoin, J. (1996). Lévy Processes. Cambridge University Press.

[4] Bertoin, J. and Doney, R. A. (1994). Cramér's estimate for Lévy processes. Statist. Prob. Lett. 21, $363-365$.

[5] Bingham, N. H., Goldie, C. M. and Teugels, J. L. (1987). Regular Variation. Cambridge University Press.

[6] Borovkov, K. ANd BurQ, Z. (2001). Kendall's identity for the first crossing time revisited. Electron. Commun. Prob. 6, 91-94.

[7] Chesney, M., Jeanblanc-Picqué, M. and Yor, M. (1997). Brownian excursions and Parisian barrier options. Adv. Appl. Prob. 29, 165-184.

[8] Dassios, A. AND Wu, S. (2009). Parisian ruin with exponential claims. Submitted. Available at http://stats. lse.ac.uk/angelos/.

[9] Dassios, A. And Wu, S. (2009). Ruin probabilities of the Parisian type for small claims. Submitted. Available at http://stats.lse.ac.uk/angelos/.

[10] Dassios, A. ANd WU, S. (2010). Perturbed Brownian motion and its application to Parisian option pricing. Finance Stoch. 14, 473-494.

[11] Dufresne, F. AND Gerber, H. (1991). Risk theory for the compound Poisson process that is perturbed by diffusion. Insurance Math. Econom. 10, 51-59.

[12] Erdélyi, A., Magnus, W., Oberhettinger, F. and Tricomi, F. G. (1954). Tables of Integral Transforms, Vol. I. McGraw-Hill, New York.

[13] Furrer, H. (1998). Risk processes perturbed by $\alpha$-stable Lévy motion. Scand. Actuarial J. 1998, 59-74.

[14] Huzak, M., Perman, M., Šikić, H. and VondračeK, Z. (2004). Ruin probabilities and decompositions for general perturbed risk process. Ann. Appl. Prob. 14, 1378-1397.

[15] Klüppelberg, C. ANd Kyprianou, A. E. (2006). On extreme ruinous behaviour of Lévy insurance risk processes. J. Appl. Prob. 43, 594-598.

[16] Klüppelberg, C., Kyprianou, A. E. and Maller, R. A. (2004). Ruin probabilities and overshoots for general Lévy insurance risk processes. Ann. Appl. Prob. 14, 1766-1801.

[17] KyPrianou, A. E. (2006). Introductory Lectures on Fluctuations of Lévy Processes with Applications. Springer, Berlin.

[18] Kyprianou, A. E. ANd Palmowski, Z. (2005). A martingale review of some fluctuation theory for spectrally negative Lévy processes. In Séminaire de Probabilités XXXVIII (Lecture Notes Math. 1857), Springer, Berlin, pp. 16-29.

[19] Landriault, D., Renaud, J. F. ANd Zhou, X. (2011). Insurance risk models with Parisian implementation delays. Submitted. Available at http://www.math.uqam.ca/ renaudjf/recherche.html.

[20] Landriault, D., Renaud, J. F. AND Zhou, X. (2011). Occupation times of spectrally negative Lévy processes with applications. Submitted. Available at http://www.math.uqam.ca/ renaudjf/recherche.html.

[21] Loeffen, R., Czarna, I. and Palmowski, Z. (2011). Parisian ruin probability for spectrally negative Lévy processes. Submitted. Available at http://arxiv.org/abs/1102.4055v1.

[22] WANG, G. (2001). A decomposition of the ruin probability for the risk process perturbed by diffusion. Insurance Math. Econom. 28, 49-59. 